derived from the 'Ac-La' preparations, have not yet been tested for their identity with cerium.

On the strength of the chemical evidence, it is difficult to avoid the conclusion that the elements denoted by the symbol ' $R \mathrm{a}$ ' in the above-mentioned disintegration scheme should in reality be designated 'Ba', and so far as the evidence has gone it seems probable that the succeeding elements 'Ac' and ' $T h$ ' will prove to be 'La' and 'Ce' respectively. It is as though, at some stage in the process resulting from the bombardment of uranium by neutrons, a 'collapse' of the nucleus occurs, giving rise to the four active isomeric alkaline-earth elements and the succeeding 'lanthanum' and 'cerium' products. The investigations are being continued.
R. W. L.

\title{
Subspecies and Varieties
}

\begin{abstract}
A DISCUSSION dealing with subspecies and varieties was held at the meeting of the Linnean Society on February 2. This discussion had been arranged at the request of the Association for the Study of Systematies in Relation to General Biology in order to obtain information as to the views and principles governing the practice of systematists in various groups of animals and plants. It was apparent from the diseussion that systematists are generally dissatisfied with the existing state of affairs. As was to be expected, little or no attention is paid to infra-specific categories in the less-known groups of both plant and animal kingdoms; but where the broader taxonomic outlines are well understood there is considerable divergence in systematic practices according to the amount of genetical, cytological and ecological work that has been done. In vertebrates, only a single infra-specific category, the 'subspecies', is generally recognized, but in entomology and botany there are others. How many there may be and their status in the taxonomic scheme are matters of dispute, and there is a disturbing confusion in the terminology applied to them. In this connexion, it may be mentioned that the Association is compiling a list of the various terms which have been used. Almost all the speakers stressed the need for more experimental work from the genetical, cytological, physiological and ecological aspects.
\end{abstract}

In opening the discussion, Mr. M. A. C. Hinton described the practice of mammalogists, who, in recent years, have used the term 'subspecies' to express stages or trends in geographical variation; they are sections of what Dr. Julian Huxley has called 'clines' (Nature, 142, 219; 1938). One principle generally accepted is that no two races of the same species can ever be found on the same ground, though exceptionally two such forms may meet after very different histories and journeys and continue to exist side by side without fusing. The principle that, unless intergradation can be demonstrated, differences, however trivial, indicate specific separation, has some theoretical support. In practice, however, it has disadvantages, especially in dealing with insular forms, and tends to mask that most interesting and instructive phenomenon, discontinuous distribution.

The definition of subspecies is an essential part of any intensive analysis of the facts of variation and distribution, and is particularly valuable in palæontology, by preventing loose identifications and consequent faulty geological deductions. It is hoped soon to commence large-scale experiments to test the values and permanence of subspecific characters and to obtain more definite information on subjects concerning which surmise alone is at present possible.
Dr. W. B. Turrill emphasized that all taxonomic categories are matters of scientific convenience based on abstractions from a continuous evolution. He recognizes that for little-known floras it is undesirable to analyse below the species level; but urged that in relatively well-studied floras the need is for intensive studies of infra-specific variation from every angle. His own researches have led him to the conclusion that taxonomic categories intergrade completely. He suggested that the term 'variety' should be used for every phenotype which is the expression of a different genotype, and that the term subspecies should be used only when a species is in process of breaking up into new ones. Names should be given to infra-specific groups only to serve some definite purpose, and in highly polymorphic species symbols might be used instead. Apomicts should receive distinct treatment.

Mr. A. J. Wilmott pointed out that confusion results from using the words species, subspecies and variety both for eategories of variation in Nature and groups of different rank in the nomenclatural system. He suggested that the terms 'binome' and 'trinome' might be substituted for species and subspecies in the nomenclatural sense, leaving the latter free for application to categories of variation. True subspecies are parts of the present time section of a lineage which has become branched by isolation, but the branches of which arenot yet completely separated. Varieties, on the other hand, are in a different category, being merely the observed phenotypic effects of separate genes, and not different kinds of organisms; they should not be given names under the same system as species and subspecies, but might be designated by symbols.

Mr. J. S. L. Gilmour dealt with the philosophical aspect of the subject and drew a distinction between taxonomic and non-taxonomic infra-specific categories. The former, which might be limited to subspecies, variety, and form, should be based so far as possible on the total attributes of the individuals concerned, while the latter should be based on a selection of attributes chosen for special purposes. Examples of such non-taxonomic categories are Dancer's 'commiscuum','comparium', and 'convivium', based on interfertility data, and useful for investigating the relationship between such data and other attributes, such as morphological differentiation. He urged that this distinetion is essential for the proper classification of infra-specific variability.

Mr.H.W.Parkershowed that the practice of herpetologists is essentially similar to that of mammalogists. $\mathrm{He}$ believes, however, that owing to lack of other data, systematists have laid undue emphasis on geographical considerations, with invidious results. Recognition by trinomials of certain forms to the 
exclusion of others equally important, and the inclusion of different grades of infra-specific rank, in the same category is misleading. The traditional methods employed in taxonomy must be supplemented for the study of infra-specific variations, and not until we know more of the categories to be dealt with can a satisfactory scheme of nomenclature be devised.

Dr. B. P. Uvarov expressed his belief that the recognition by entomologists of subspecies, in the sense of geographical races, has been of great practical convenience; it has enabled taxonomists to unite numerous minor forms into large species without losing sight of their individuality. Recent physiological, ecological and genetical work has revealed the existence of races which are indistinguishable by external characters, Between these incipient, deeply seated, internal changes and the appearance of external morphological characters there is probably a time lag; consequently even the smallest external differences, if not irregular and obviously casual, deserve the closest attention as representing quite an advanced stage in variation.

Prof. MeClung remarked that the perfect taxonomy has been described as the summation of all biological knowledge ; we have obviously not yet reached perfection and the present discontent shows a striving in the right direction.

Mr. C. E. Hubbard said that the terms subspecies and variety are used by systematic botanists to cover every grade from a slight variation in one character to a well-defined species. In the floras of large tropical areas there occur numerous polymorphic species which, in the absence of detailed knowledge, it seems best to lump together and note the polymorphism. Where variations are of economic importance, those that are well defined can be given binary names with the view of making reference easier.

Prof. F. E. Weiss agreed with the previous speakers that investigations of the taxonomic categories need further biological knowledge, especially genetical. Frequently genetical analysis can establish the status of a taxonomic group when the systematist is in doubt. A good example is seen in the pimpernels; of the two blue forms, one has proved to be a genetically stable species and the other, scarcely distinguishable by any other criteria, merely a mutation of the red species.

Dr. Julian Huxley emphasized that taxonomy deals with natural groups having a real existence. Discussing infra-specific variability, he pointed out that in the gradation between varieties, subspecies and species, the points of stability are more frequent than the intergrading zones. He dealt with various causes of discontinuity and mentioned especially isolation leading to non-adaptive variation, and the production of partial discontinuity by the formation of internally stable gene-complexes. For the description of more or less continuous variation he suggested the use of his 'cline' concept.

Dr. R. Melville pointed out that hybridization is much commoner in plants than in animals and that in certain groups the species can be arranged on an intergrading series corresponding to a 'cline' in Huxley's sense, but with no geographical basis. $\mathrm{He}$ suggested that new terminology should be used for forms constituting these series.

Prof. W. Garstang commented on the difficulties of genetical research on animals, and directed attention to the existence of 'clines' in fishes, particularly herrings and mackerel. In some instances, the 'clines' can be correlated with gradual environmental changes.

\section{Interstellar Space}

$\mathrm{O}^{\mathrm{N}}$ May 13, 1938, Prof. Charles Fabry delivered the George Darwin Lecture at the Royal Astronomical Society and dealt with the problem of the nature of the matter which exists in interstellar space (Mon. Not. Roy. Astro. Soc., 98, 9, Supp. No., October 1938). The subject is so vast that it was possible for Prof. Fabry only to review briefly the different methods of investigation and the interpretation which has been placed upon what they reveal. As is well known, the Doppler effect has enabled the interstellar lines to be detected. The displacement of the lines in stellar spectra gives the radial velocity and this should be the same for all lines. If a line gives a velocity which differs from that derived from the displacements of other lines, the discrepancy must be due to matter situated between the star and ourselves-in other words, interstellar matter. In 1904, Hartmann observed the spectrum of $\delta$ Orionis, a star of variable radial velocity, and found that the velocity, measured on the hydrogen lines, varied between +133 and $-66 \mathrm{~km}$./sec., but the lines of ionized calcium showed a constant velocity of $+16 \mathrm{~km}$. $/ \mathrm{sec}$. This supplied the evidence for the existence of a cloud of ionized calcium between the star and ourselves. It seems remarkable that for fifteen years these ionized calcium lines were the only ones which were known with any certainty. In 1919, however, Miss Heger established without doubt the existence of neutral lines of sodium, and with improvements in spectroscopic methods more interstellar lines have been discovered. It is interesting to notice that the exploration of the ultra-violet lines of sodium by Adams and Dunham has been rendered possible by the use of aluminized mirrors and of gratings ruled on an aluminized layer. The discovery of other lines which cannot be identified has caused some speculation regarding their origin, and Prof. Fabry conjectures that microscopic crystals, perhaps giving absorption bands at the very low temperature, may be responsible for some of them.

Among some of the interesting results accomplished may be mentioned the estimate of the mean radial velocity of the interstellar matter between the star and ourselves, and it seems that the interstellar gas has the same mean rotation as the galaxy. Quite recently, however, Beals has shown that in some cases it is possible to detect masses of interposed gas which have different velocities. Important developments may be expected in this particular sphere.

It is impossible in the limited space to refer to more than a few of the points dealt with in the lecture. 